\title{
Secreted Clusterin Gene Silencing Enhances Chemosensitivity of A549 Cells to Cisplatin Through AKT and ERK1/2 Pathways in Vitro
}

\author{
Biyuan Zhang ${ }^{\mathrm{a}}$ Kejun Zhang ${ }^{\mathrm{b}}$ Zimin Liu ${ }^{\mathrm{a}}$ Fengyun Hao ${ }^{c}$ Mei Wangd,e \\ Xiangchun Lij ${ }^{a, e}$ Zongsiu Yin ${ }^{a, e}$ Hui Liang $^{f}$ \\ IInternal Medicine-Oncology, ${ }^{b}$ Surgery, ${ }^{\text {CPathology, }}{ }^{d}$ Respiratory Medicine, the Affiliated Hospital \\ of Medical College, Qingdao University, Qingdao, ${ }^{e}$ Center Laboratory, the Zhongshan Hospital of

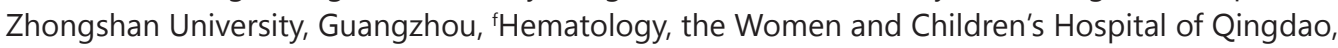 \\ Qingdao, China
}

\section{Key Words}

Lung cancer • Chemotherapy • Clusterin • Cisplatin • AKT • ERK1/2

\begin{abstract}
Background/Aims: Several studies have shown secreted clusterin ( $\mathrm{SCLU}$ ) silencing directed against sCLU mRNA in sCLU-rich lung cancer cell lines sensitized cells to chemotherapy. However, the molecular mechanisms underlying the effect of sCLU silencing on lung cancer cell chemosensitivity is not known. In the present study, we aimed to determine that vector expressing short hairpin RNA against sCLU RNA (sCLU-shRNA) enhances the chemosensitivity in human small cell lung cancer A549 cells in vitro by inhibition of phosphorylated ERK1/2 ( $p$-ERK1/2) and Akt (p-Akt). Methods: The pCDNA3.1-sCLU and control scrambled pCDNA3.1 plasmid was constructed. We investigated the effects of sCLU overexpression by pCDNA3.1sCLU transfection on chemosensitivity to cisplatin (DDP) in A549 cells in vitro. We downregulated SCLU expression by short hairpin RNA against SCLU RNA (sCLU-shRNA) and investigated the effects on chemosensitivity to DDP in A549 cells and A549DDP in vitro. In order to confirm the correlation between SCLU and AKT and ERK1/2 signals, cells were treated with wortmannin and U0126. Results: We found the chemotherapeutic agent DDP activated SCLU. Overexpression of SCLU increased cellular DDP chemoresistance in the A549DDP and pCDNA3. 1-sCLU transfected A549 cells via inhibition DDP-induced apoptosis. Whereas sCLU knockdown induced chemosensitization in the S549 and A549DDP cells via increase of DDPinduced apoptosis. sCLU overexpression activated pAKT Ser ${ }^{473}$ and pERK1/2 ${ }^{\text {Thr202/Tyr204 }}$, and vice versa. Inhibition of $\mathrm{pAKT}$ Ser ${ }^{473}$ and $\mathrm{pERK1} / 2^{\mathrm{Th}}{ }^{202 / \mathrm{Ty} r 204}$ was sufficient to induce significant recovery in chemosensitivity to DDP in $A 549^{D D P}$ in the presence of $S C L U$ overexpression. The DDP activated sCLU, which directly regulated pAKT and pERK1/2. Conclusions: This novel finding suggests that therapies directed against SCLU and its downstream signaling targets PAKT and pERK1/2 may have the potential to enhance the efficacy of DDP-based chemotherapy.
\end{abstract}

Copyright $@ 2014$ S. Karger AG, Basel 


\section{Introduction}

Lung cancer, the leading cause of cancer death worldwide, can be divided into two types: non-small cell lung cancer (NSCLC) and small cell lung cancer (SCLC). NSCLC comprises $>75 \%$ of lung cancer cases making it the leading cause of cancer-related death. The prognosis for NSCLC patients is highly dependent on the stage at diagnosis, and despite efforts to develop early screening tools, a majority of tumors are detected at an advanced stage [1]. The therapies available to date for NSCLC treatment are surgery, radiotherapy, and chemotherapy. Because of the size and distribution of lung cancer, the cytoreductive surgery is not very effective for this disease and therefore chemotherapy and/or radiation are the only treatments of choice. Despite major advances in patient management, chemotherapy and radiotherapy, nearly $80 \%$ of the patients still die within one year of diagnosis and longterm survival is obtained only in $5-10 \%$ of the cases [2]. Chemoresistance occurs not only to clinically established therapeutic agents but also to novel targeted therapeutics. However, extensive stage disease has initial response rates to chemotherapy exceeding $70 \%$, the disease almost invariably progresses and becomes fatal [3]. Both intrinsic and acquired mechanisms have been implicated in drug resistance but it remains controversial which mechanisms are responsible that lead to failure of therapy in cancer patients [4-6].

Clusterin is an enigmatic glycoprotein with a nearly ubiquitous tissue distribution. It plays important roles in various pathophysiological processes, including tissue remodeling, reproduction, lipid transport, complement regulation, and apoptosis. Clusterin appears to have two main isoforms that result from alternative splicing. The secreted and nuclear forms of clusterin have been reported to play different roles in human malignancies[7]. The secreted form of the CLU protein (sCLU) is a glycosylated protein of 76-80 kDa. Upregulation of sCLU mRNA and protein levels detected in diverse disease states and in vitro systems have led to suggestions that it functions in membrane lipid recycling, in apoptotic cell death, and as a stress-induced secreted chaperone protein, amongst others[8].

It has found sCLU was overexpressed in a number of malignant tumors and has been proven to correlate closely with the chemoresistance of several cancer cells to chemotherapeutic agents [9-15]. Several in vitro and vivo studies have shown sCLU silencing by antisense oligonucleotides (ASO) and small-interfering RNAs (siRNA) directed against sCLU mRNA in sCLU-rich lung cancer cell lines sensitized cells to chemotherapy and radiotherapy and decreased their metastatic potential [16-20]. Studies performed in lung cancer cell lines and animal models showed that sCLU is upregulated after exposure to chemo- $[19,21]$ and radiotherapy [20]. A potential role proposed for the protein is cytoprotective. However, the molecular mechanisms underlying the effect of sCLU overexpression on chemoresistance and cytoprotection in NSCLC is not known.

Activation of the phosphoinositide 3 kinase (PI3K)/AKT signalling pathway has been linked with resistance to chemotherapeutic drugs, and its downregulation, by means of PI3K inhibitors, lowers resistance to various types of therapy in tumour cell lines [22-24]. Mitogen-activated protein kinase/extracellular signal regulated kinase (ERK) pathway plays an essential role in the development and progression of various tumors. ERK1/2 is a key component of this pathway. The hyperactivation of ERK1/2 also has been shown to promote resistance to chemotherapy drugs in many cancer cells [25-27]. Inhibiting the action of ERK1/2 prevents tumor cell proliferation, promote apoptosis and reverse resistance to therapy $[28,29]$.

In lung cancer, phosphorylated ERK1/2 (pERK1/2) and AKT (pAKT) can be further stimulated by chemotherapeutics, and targeting the ERK1/2 or AKT pathway has been reported to sensitize cancer cells to therapy [30-32]. We have also recently found ERK1/2 and AKT pathways contribute to cisplatin resistance in human small cell lung cancer A549 cells, and blocking these pathways may be an effective strategy for improving the efficacy of cisplatin as anticancer treatment [33]. Tang et al. [28] has found knockdown of clusterin sensitizes pancreatic cancer cells to gemcitabine by inhibition of gemcitabine-induced clusterin-pERK1/2 activation. In breast cancer, clusterin may regulate the aggressive 
behaviour of human breast cancer cells through modulation of ERK1/2 signalling and MMP9 expression [34]. In lung cancer cells, clusterin regulate EMT and aggressive behaviour through modulating ERK1/2 signalling and Slug expression [16].We therefore suggested ERK1/2 signalling is regulated by clusterin. Many studies in literature have reported that AKT pathway may also regulated by clusterin in cancers including lung cancer $[17,35,36]$.

Given the importance of clusterin in regulation of AKT and ERK1/2 signaling, we hypothesized that targeting clusterin might represent a novel approach to modulate the chemoresistance of lung cancer cells to cisplatin. We present the first evidence indicating a role for clusterin in determining lung cancer cellular chemoresistance to cisplatin, the mechanism by which is via regulating AKT and ERK1/2 signaling.

\section{Materials and Methods}

\section{Cell Lines}

Human lung adenocarcinoma bronchioloalveolar carcinoma A549 cells and cisplatin (DDP) resistant A549 cells (A549DDP) were obtained from the American Type Culture Collection (Manassas, VA) and cultured at $37^{\circ} \mathrm{C}$ in a humidified atmosphere containing $5 \% \mathrm{CO}_{2}$ in Ham's F12 medium supplemented with sodium bicarbonate $(2.2 \%, \mathrm{w} / \mathrm{v})$, L-glutamine $(0.03 \%, \mathrm{w} / \mathrm{v})$, penicillin $(100 \mathrm{units} / \mathrm{ml})$, streptomycin $\left(100 \mu \mathrm{g} \mathrm{ml}^{-1}\right)$, and fetal calf serum $(10 \%)$.

\section{Reagents}

Akt (Ab-473) antibody (E021054-2), ERK1/2 (Ab-202/204) Antibody (E022017-2), ERK1/2 (PhosphoThr ${ }^{202 / T y r 204}$ ) Antibody (E012017-2), AKT (Phospho-Ser ${ }^{473}$ ) Antibody (E011054-2) and $\beta$-actin Antibody(5B7) (E12-041-3) were purchased from EnoGene,Shanghai,China. Clusterin (A-9)(sc-166907,1:200) was purchased from Santa Cruz,Shanghai,China. Horseradish peroxidase-conjugated goat anti-mouse and donkey anti-rabbit antibodies were obtained from Jackson ImmunoResearch (West Grove, PA). The enhanced chemiluminescence detection kit was from Amersham (Piscataway, NJ). DDP was purchased from Sigma (St. Louis, MO). Wortmannin [the inhibitor of phosphatidylinositol 3-kinase (PI3K)] (1670-1) and U0126 which is a selective inhibitor of MEK-1/2 (ERK1/2 upstream kinase) were purchased from Biovision, Beijing, China. A stock solution of U0126 (10 mmol/L) was prepared using DMSO as the solvent.

Full-length human clusterin cDNA expression vectors

Reverse transcription-PCR of normal human fibroblast total RNA was done using the primers 5'-GACTCCAGAATTGGAGGCATG-3' (forward) and 5'-ATCTCACTCCTCCCGGTGCT-3' (reverse) [37]. The cDNA was cloned into pCR ${ }^{\mathrm{TM}}$ II (INVITROGEN). sCLU full-length cDNA was then subcloned into the pCDNA3.1. to produce the pCDNA3.1-sCLU vector. Constructs have been sequenced before carrying out expression experiments.

\section{pCDNA3.1-sCLU transfections}

Total of $5 \times 10^{5}$ A549 cells were plated in 6-cm dishes and transfected using the Lipofectamine 2000 (Invitrogen), according to the manufacturer's protocol. A549 cells were transfected with endotoxin-free preparations of pcDNA3.1-sCLU or pcDNA3.1 (control). Transiently transfected cells were harvested 48 hs after transfection. Transfection efficiency was assessed by detection of green fluorescence protein (GFP) expression by fluorescence microscopy. Positively transfected cells were routinely more than $50 \%$ of the cell population. For selection of stably transfected cell populations, G418 was added to the culture medium $48 \mathrm{hs}$ after transfection at a concentration of $400 \mu \mathrm{g} \mathrm{ml}^{-1}$ for A549 cells. The cells were selected with $400 \mu \mathrm{g}$ $\mathrm{ml}^{-1} \mathrm{G} 418$ for $14 \mathrm{ds}$. Selected colonies were screened by immunoblotting to identify stable clones expressing sCLU.

\section{Short hairpin RNA transfection}

Vector expressing short hairpin RNA against sCLU RNA (sCLU-shRNA) plasmid and control scrambled plasmid were purchased from Santa Cruz (Shanghai,China). A549DDP cells were transfected with sCLUshRNA or control scrambled using the Lipofectamine 2000 according to the manufacturer's protocol. G418 was added to the culture medium 48 hs after transfection at a concentration of $600 \mu \mathrm{g} \mathrm{ml}^{-1}$ for A549 cells. 
The cells were selected with $600 \mu \mathrm{g} \mathrm{ml}^{-1} \mathrm{G} 418$ for $14 \mathrm{ds}$ to acquire the stably transfected A549DDP/sCLUshRNA. The knockdown effect was verified by Western Blotting analysis using anti-sCLU antibodies.

\section{Drug Treatments}

DDP was purchased from Sigma-Aldrich Co. Dimethyl sulfoxide was used to dilute cisplatin to a 20 mM stock. A549, A549DDP, A549/pCDNA3.1-sCLU, A549DDP/sCLU-shRNA, A549/pCDNA3.1 and A549DDP/ pCDNA3.1 cells were treated with cisplatin $(1-50 \mu \mathrm{M})$ for $72 \mathrm{hs}$ or with the same concentration of DDP for the same duration followed by wortmannin ( $400 \mathrm{nM}$ ) for $4 \mathrm{~h}$ or U0126 (25 uM) for $8 \mathrm{hs}$ in the continuous presence of DDP. The concentration and duration of DDP treatment were chosen based on preliminary studies examining its effects on cell growth inhibition and induction of apoptosis.

\section{Western blot analysis}

For Western blot analysis, cells or xenograft tissues were rinsed in ice-cold PBS twice and lysed in cell lysis buffer $(10 \mathrm{mM}$ Tris- $\mathrm{HCl}, \mathrm{pH} 7.4,150 \mathrm{mM} \mathrm{NaCl}, 1 \%$ sodium deoxycholate, $1 \%$ Nonidet P-40, 1 $\mu \mathrm{g} / \mathrm{ml}$ aprotinin, $1 \mu \mathrm{g} / \mathrm{ml}$ leupeptin, $50 \mathrm{mM} \mathrm{NaF}, 2 \mathrm{mM} \mathrm{Na} 3$ V04, $2 \mathrm{mM}$ EGTA, $2 \mathrm{mM}$ EDTA, and $0.25 \mathrm{mM}$ PMSF) for $30 \mathrm{~min}$. Samples were sonicated for $30 \mathrm{~s}$ and centrifuged for $20 \mathrm{~min}$ at $12,000 \times \mathrm{g}$ at $4^{\circ} \mathrm{C}$. Samples were electrophoresed on a $12 \%$ SDS-polyacrylamide gel and electrophoretically blotted on nitrocellulose membrane. Membranes were blocked in TBS/Tween-20 with 5\% milk and incubated with primary Abs diluted in TBS/Tween-20/BSA overnight at $4{ }^{\circ} \mathrm{C}$. The following primary antibodies were used: $\beta$-actin $(1$ : 10000), sCLU(1:200), AKT (Ab-473)(1:100), ERK1/2 $\left(\mathrm{Ab}^{-202 / 204}\right)(1: 200)$, ERK1/2 (Phospho-Thr202/Tyr204) (1:200) and AKT (Phospho-Ser473)(1:100). All blots were incubated with secondary Abs conjugated to HRP $(1 / 2000)$ and developed using the ECL method. Protein concentration was determined using bicinchoninic acid assay (Pierce, Rockford, IL).

\section{Cell viability assay}

Cell viability was evaluated using cell counting kit (CCK-8). Briefly, A549, A549DDP, A549/pCDNA3.1sCLU, A549DDP/sCLU-shRNA, A549/pCDNA3.1 and A549DP/pCDNA3.1 cells were pre-cultured in 96well plate $(3,000$ cells/well) for 24 hs. 72 hs after DDP treatment at the indicated doses, culture media were replaced by the WST-8 reagent. Reduced WST-8 by the cellular dehydrogenases turns into orange formazan. Produced formazan is directly proportional to living cells. Absorbance was measured at $450 \mathrm{~nm}$ by microplate reader equipped by computer.

\section{Determination of apoptotic cells by FACS analysis}

Quantification of apoptosis analysis was carried out as the manufacture's instruction. In brief, following treatment, the cells were collected by gentle trypsinisation, washed in phosphate-buffered saline (PBS), and pelleted by centrifugation. The cells were fixed in $70 \%$ ethanol,washed twice in PBS and resuspended in PBS containing RNAse A $\left(20 \mathrm{mgml}^{-1}\right.$ ). The cells were stained with PI (final concentration $0.1 \mathrm{mg} \mathrm{ml}^{-1}$ ) for 10 $\mathrm{min}$ at room temperature. The samples underwent FACS analysis (FL-3 channel) using a Beckman Coulter Counter Epics XL flow cytometer. For each sample, 10000 events were collected and stored for subsequent analysis using EXPO software. Data were elaborated using Autofit feature of the Multicycle for Windows software and expressed as fraction of cells in the different cycle phases. The percentage of cells in the sub-G0 phase was quantitated as an estimate of cells undergoing apoptosis.

\section{Terminal dUTP nick-end labeling (TUNEL) assay}

Fixed cells were incubated with an equilibrium buffer for 5 min using the in situ apoptosis detection kit. Apoptosis of the cells was evaluated on the basis of the TUNEL assay according to the manufacturer's instructions. For quantifying apoptotic cells, apoptotic and total cells were counted in 5 random fields scoring between 300 and 500 cells, and the numbers of apoptotic cells were expressed as percentages of the total cell population. TUNEL staining slides were observed with microscope. All assays were performed in quadruplicate.

\section{Statistical analysis}

Student's $t$-test was used to compare mean values where appropriate using SPSS11.0 software. $P$ values $<0.05$ were considered significant. All data are expressed as means \pm SD for a series of experiments. 


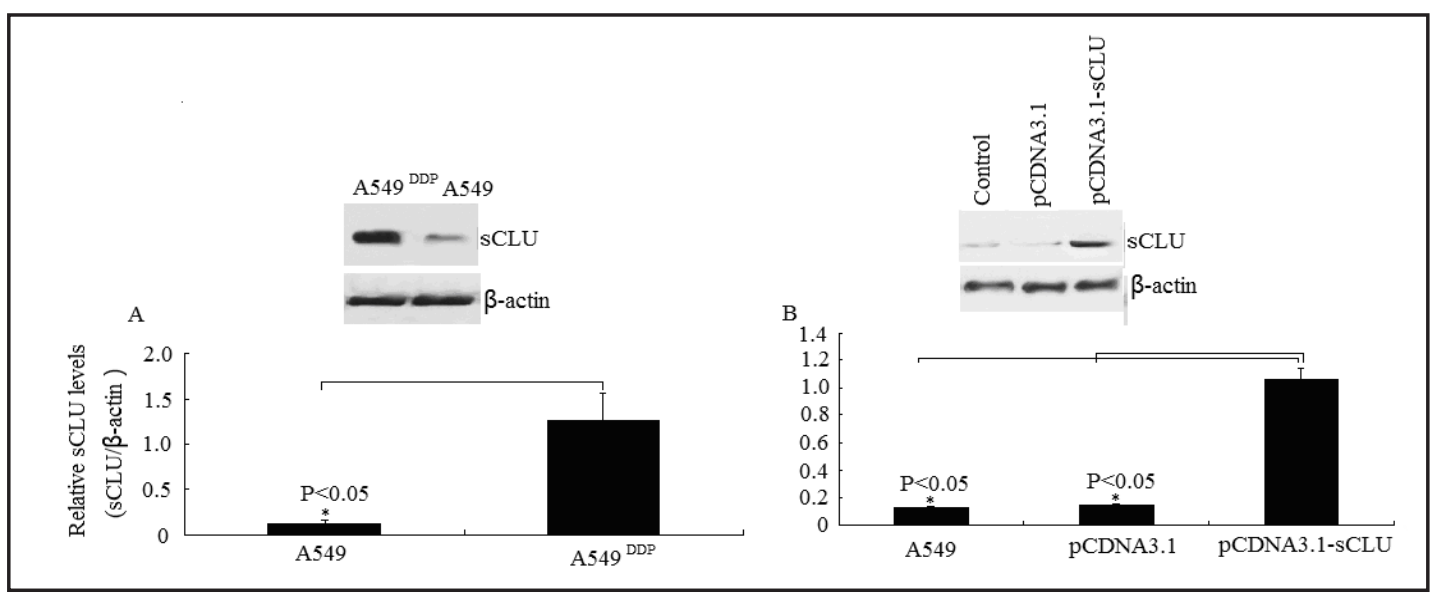

Fig. 1. Effective sCLU expression in A549 cells after pCDNA3.1-sCLU transfection. A, sCLU protein expression was detected in A549 ${ }^{\text {DDP }}$ cells by western blot assay. B, sCLU protein expression was detected in pCDNA3.1sCLU and scrambled pCDNA3.1 transfected A549 cells by western blot assay.

Fig. 2. Effective silencing of the sCLU expression in A549 ${ }^{\mathrm{DDP}}$ cells after shRNA transfection. sCLU expression was knockdown by western blot assay after stably shRNA of sCLU transfection.

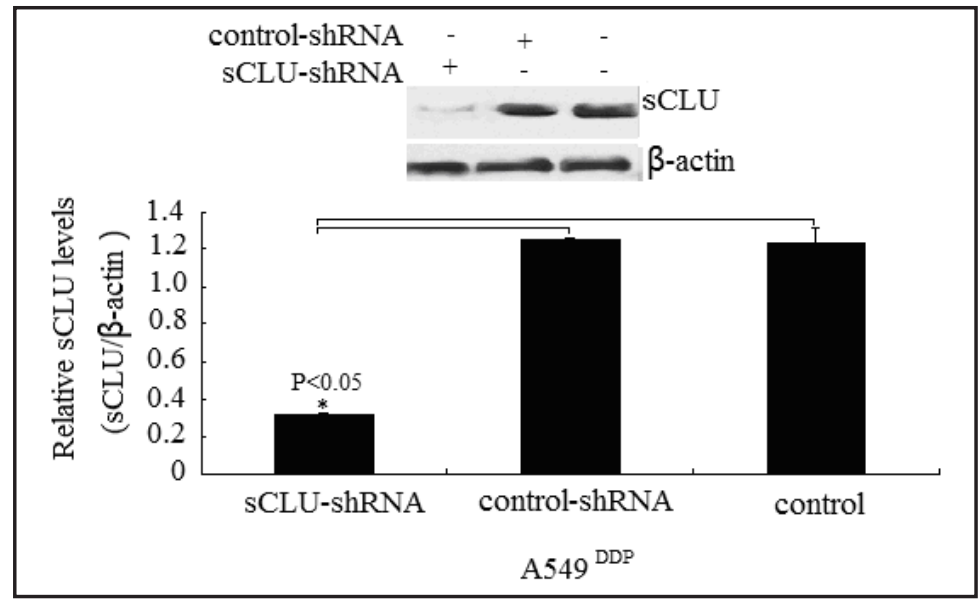

\section{Results}

sCLU was overexpressed in DDP resistant A549 cells (A549DDP)

As shown in Figure 1A, sCLU protein was overexpressed in DDP resistant A549 cells $\left(A 549^{\mathrm{DDP}}\right)(1.26 \pm 0.21)$ compared to the 4549 cells $(0.12 \pm 0.03)(P<0.05$, Fig. $1 \mathrm{~A})$.

Successful overexpression of sCLU through pCDNA3.1-sCLU transfection in A549 cells

Fluorescence microscopy was used to detect the pCDNA3.1-sCLU transfection efficiency in A549 cells. After 48 hs transfection, the green staining of more than $75 \%$ of cells was confirmed in pCDNA3.1 transfected A549 cells.

In the pCDNA3.1-sCLU clones, expression of exogenous sCLU was significantly higher compared to the endogenous sCLU levels in the controls (pCDNA3.1). As shown in Figure 1B, sCLU protein was overexpressed in pCDNA3.1-sCLU transfected A549 cells (1.06 \pm 0.24$)$ compared to the A549 cells $(0.12 \pm 0.03)$ and control pCDNA3.1 transfected A549 cells $(0.14 \pm 0.03)(P<0.05)$.

Efficient silencing of sCLU expression in A549DDP cells using shRNA transfection against $s C L U$

A549DDP cells was stably transfected with scrambled control or shRNA of sCLU. Protein expression was evaluated in whole-cell extracts from A549 ${ }^{\mathrm{DDP}}$ cells. Comparing to the scrambled control, sCLU knockdown was shown to be successful across the A549 ${ }^{\text {DDP }}$ cells 


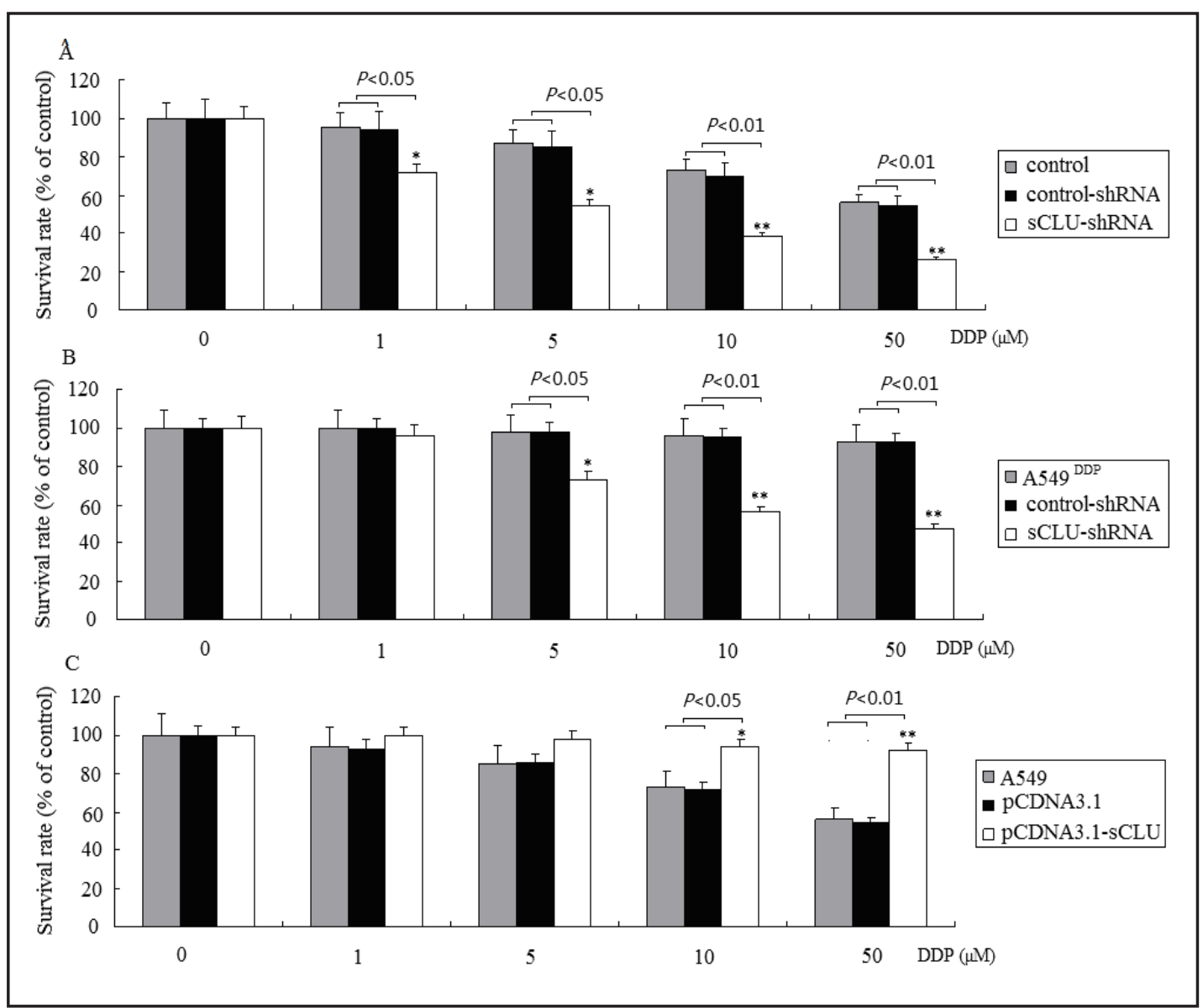

Fig. 3. sCLU level is associated with DDP sensitivity in A549 cells. A, A549/shRNA or A549/sCLU-shRNA stable clone were seeded into 96-well plates. The following day, 1-50 $\mu \mathrm{M}$ DDP were applied. After incubation for $72 \mathrm{hs}$, cell survival was analyzed by MTT. Cell growth in the absence of DDP corresponds to 100. B, A549 ${ }^{\mathrm{DDP}}$ was stably transfected with control or sCLU shRNA, then treated with 1-50 $\mu \mathrm{M}$ cisplatin for $72 \mathrm{hs.}$ MTT assays were performed the cell survival. C, A549 cells was stably transfected with pCDNA3.1 control or pCDNA3.1-sCLU, then treated with 1-50 $\mu \mathrm{M}$ cisplatin for $72 \mathrm{hs}$. MTT assays were performed the cell survival. Boxes, mean; bars, \pm SD.

(Fig. 2). Furthermore, sCLU mRNA gene expression was also inhibited after stably shRNA of sCLU transfection (data not shown).

\section{sCLU silencing inhibits DDP-induced growth in A549 cells}

To characterize the role of sCLU in DDP resistance, A549 cells were stablly transfected with control vector or SCLU-shRNA vector against sCLU to generate A549/shRNA or A549/ sCLU-shRNA stable clone, respectively (Fig. 2). The A549/shRNA or A549/sCLU-shRNA stable clone were treated with 1-50 $\mu \mathrm{M}$ cisplatin for $72 \mathrm{hs}$. Cytotoxicity of DDP in control and sCLU knockdown cells were measured by MTT assays. As shown in Figure 3A, A549 cells depleted of sCLU displayed decreased cell survival after DDP treatment for $72 \mathrm{hs,} \mathrm{compared}$ with those of control cells and control-shRNA transfected A549 cells. sCLU-shRNA or shRNA transfection alone did not affect the survival after DDP treatment (data not shown)

To examine whether depletion of sCLU can re-sensitize DDP resistant A549 ${ }^{\text {DDP }}$ cells, A549 ${ }^{\text {DDP }}$ was stably transfected with control or sCLU shRNA to establish control and sCLU knockdown cell lines (Fig. 2). As shown in Figure 3B, depletion of sCLU in A549DDP cells dramatically decreased the survival of DDP in sCLU knockdown cells measured by MTT assays. 


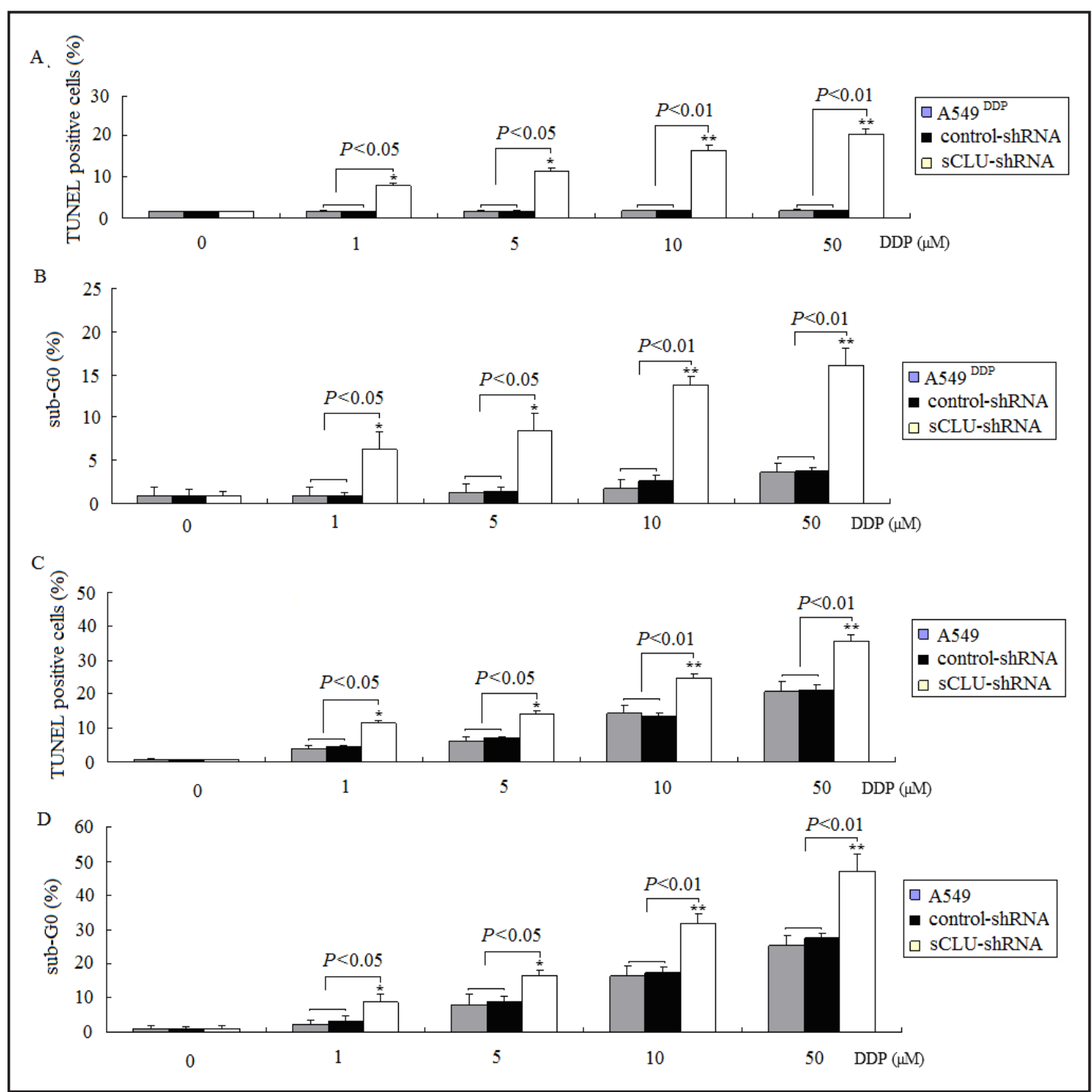

Fig. 4. sCLU silencing on DDP-induced apoptosis in A549 cells. A549DDP cells, A549DDP cells stably transfected with control or sCLU were treated with 1-50 $\mu \mathrm{M}$ cisplatin for $72 \mathrm{hs}$. Apoptosis was analyzed by TUNEL analysis (A) and FACS assay(B). A549 cells and A549/sCLU-shRNA cells were treated with 1-50 $\mu$ M DDP for 72 hs. Apoptosis was analyzed by TUNEL analysis (C) and FACS assay (D). Boxes, mean; bars, \pm SD. ${ }^{*}, P<$ $0.05,{ }^{* *}, P<0.01$ (compared with control or cells transfected with mock expression plasmid).

sCLU overexpression confers resistance to DDP in vitro in A549 cells

To examine whether sCLU overexpression can be resistant to DDP treatment, A549 cells was stably transfected with pCDNA3.1 control or pCDNA3.1-sCLU to establish control and sCLU overexpressed A549 cells (Fig. 1C). As shown in Figure 3C, overexpression of sCLU in A549 cells dramatically decreased cell survival of DDP in sCLU overexpressed cells measured by MTT assays. pCDNA3.1 control or pCDNA3.1-sCLU transfection alone did not affect the survival after DDP treatment (data not shown). The data presented above suggest that stable overexpression of sCLU or stable silencing of sCLU alone did not affact cell survival of the A549 cells, but sCLU confers DDP resistance.

\section{sCLU silencing increases DDP-induced apoptosis in A549 cells}

In addition to testing apoptosis levels of sCLU silencing on DDP induced apoptosis, the A549 ${ }^{\text {DDP }}$ cells, sCLU-shRNA stably transfected A549 cells and their controls were chosen for study. 


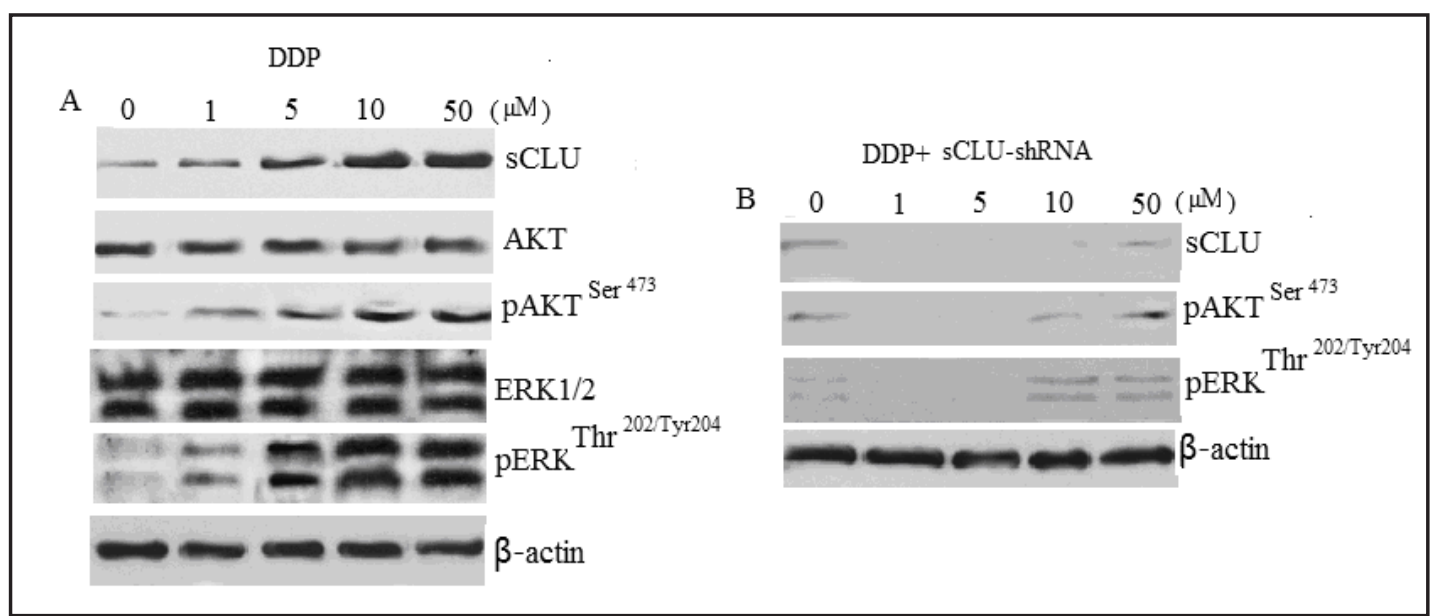

Fig. 5. Regulation of DDP-induced pERK1/2 and pAKT upregulation by sCLU. A, A549 cells were treated with DDP (1-50 $\mu \mathrm{M})$ for 72 hs. Protein expression was evaluated in whole-cell extracts from A549 cells by Western blotting. B, A549 cells were stably transfected with sCLU-shRNA against sCLU, then treated with 1-50 $\mathrm{M}$ DDP for 72 hs. Protein expression was evaluated in whole-cell extracts from A549 cells by western blotting.

We first analyzed the sCLU alone on apoptosis in A549 cells. A549 cells were transiently transfected with sCLU-shRNA, and its controls for 72 hs, no significant apoptotic cells was increased by TUNEL staining and FACS assay (data not shown).

To analyze the extent of the DDP-mediated cell death, we scored apoptosis by TUNEL and FACS. A549DDP cells were treated with 1-50 $\mu \mathrm{M}$ cisplatin for $72 \mathrm{hs}$, no significant TUNEL positive cells were shown. To observe whether depletion of sCLU can re-sensitize DDP resistant A549 ${ }^{\text {DDP }}$ cells, A549 ${ }^{\text {DDP }}$ was stably transfected with control or sCLU shRNA to establish control and sCLU knockdown cell lines (Fig. 2). The results showed depletion of sCLU in A549DDP cells dramatically increased TUNEL positive cells in the A549 ${ }^{\text {DDP }} /$ sCLU shRNA cells (Fig. 4A). Similar results were also shown by FACS assay (Fig. 4B).

A549 cells treated with 1-50 $\mu \mathrm{M}$ cisplatin for 72 hs induced a few apoptotic cells, however, in the sCLU-shRNA stably transfected A549 cells, treated with 1-50 $\mu \mathrm{M}$ cisplatin for 72 hs induced much more TUNEL positive cells (Fig. 4B). Similar results were also shown by FACS assay (Fig. 4D). These results above are consistent with cell growth inhibition studies by MTT, suggesting that the loss of viable cells by sCLU-shRNA transfection is partly due to the induction of an apoptotic cell death mechanism.

DDP activates sCLU and SCLU-dependent pERK1/2 and pAKT in A549 cells

A549 cells were treated with DDP (1-50 $\mu \mathrm{M})$ for 72 hs. Protein expression was evaluated in whole-cell extracts from A549 cells by western blotting. As shown in Figure 5A, sCLU, pERK1/2 and pAKT protein expression was elevated in A549 cells after 1,5,10 and $50 \mu \mathrm{M}$ DDP treatment for 72 hs.

To determine if pERK1/2 and pAKT protein expression was sCLU dependent, A549 cells were stably transfected with control vector or sCLU-shRNA vector against sCLU, then treated with 1-50 $\mu \mathrm{M}$ cisplatin for $72 \mathrm{hs}$. Western blot results shown no statistical sCLU, pERK1/2 and pAKT protein expression upregulation was found in SCLU-shRNA vector transfected cells compared to the cells treated with DDP alone (Fig. 5B) or control transfected cells treated with DDP (data not shown).

When the A549 cells were treated with wortmannin $(400 \mathrm{nM})$ for 4 hs or U0126 $(25 \mu \mathrm{M})$ for $8 \mathrm{hs}$, then treated with 1,5,10 and $50 \mu \mathrm{M}$ cisplatin for $72 \mathrm{hs}$, pERK1/2 and pAKT protein expression was inhibited. However, sCLU was activated in the continuous presence of DDP all 


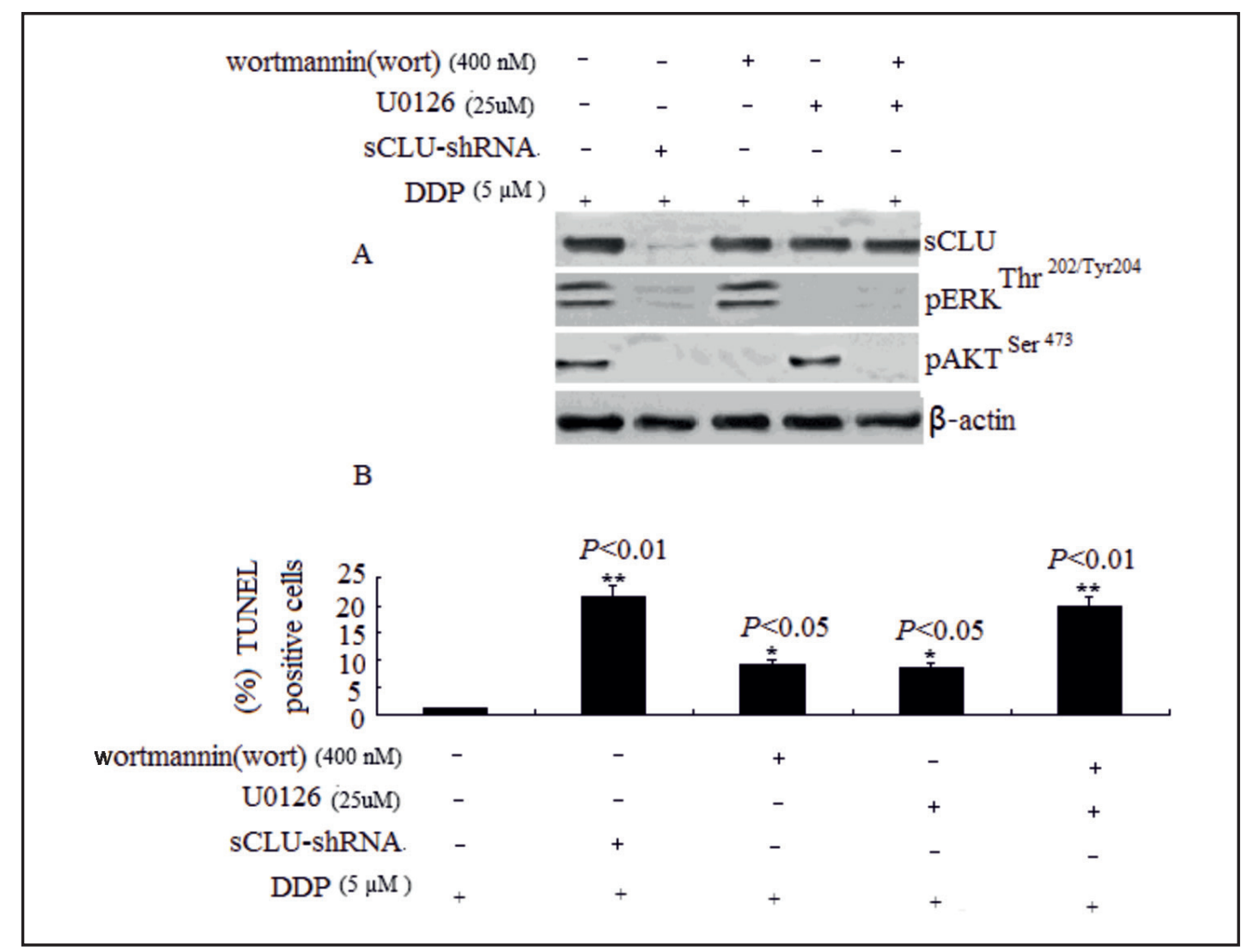

Fig. 6. sCLU silencing sensitizes A549 ${ }^{\mathrm{DDP}}$ cells to DDP via sCLU-dependent inhibition of pERK1/2 and pAKT. $A$, sCLU-shRNA stably transfected A549 ${ }^{\text {DDP }}$ cells was treated with $5 \mu \mathrm{M}$ DDP for 72 hs, A549 ${ }^{\text {DDP }}$ cells was treated with DDP combined with wortmannin ( $400 \mathrm{nM})$ or U $0126(25 \mu \mathrm{M})$,or treated with DDP and in combination with wortmannin and U0126. Western blot analysis of sCLU,pERK1/2 and pAKT in different groups. $B$, Apoptosis was analyzed by TUNEL staining in different groups. Boxes, mean; bars, \pm SD. ${ }^{*} P<0.05,{ }^{* *}, P<0.01$ (compared with A549 ${ }^{\text {DDP }}$ control).

the same (data not shown). These results demonstrate that DDP activates SCLU -dependent pERK1/2 and pAKT expression in A549 cells.

sCLU silencing sensitizes A549 cells to DDP via inactivtion of $p E R K 1 / 2$ and $p A K T$

To determine the biological significance of the functional link between clusterin and AKT and ERK, we have evaluated the effects of sCLU knockdown DDP induced A549 cell death. As shown in Figure 6A, A549DDP has higher sCLU levels, the pERK1/2 and pAKT was also activated in the A549DDP cells. In A549DDP /sCLU-shRNA cells, the sCLU, pERK1/2 and pAKT was specifically silenced. In contrast, inhibition of pERK1/2 and pAKT by wortmannin or/and U 0126 did not affect sCLU levels in the A549DDP cells, suggesting that ERK1/2 and AKT is a critical downstream mediator of sCLU.

It has shown above that A549 ${ }^{\mathrm{DDP}}$ cells depleted of sCLU displayed decreased cell survival and increased apoptotic cells after DDP treatment for $72 \mathrm{hs,} \mathrm{compared} \mathrm{with} \mathrm{those} \mathrm{of} \mathrm{control}$ cells and control-shRNA transfected A549 cells.

We then investigated if concurrent blockage of pERK1/2 and pAkt cooperatively potentiates DDP $(5 \mu \mathrm{M})$-induced cancer A549 ${ }^{\mathrm{DDP}}$ cells death and survival inhibition. A549 ${ }^{\mathrm{DDP}}$ cells were treated with wortmannin or U0126 alone or combined wortmannin and U0126 to simultaneously block pAKT and pERK1/2, and examined the effect of which on DDP -induced cancer cell apoptosis and survival inhibition. 
Treatment with DDP caused no detectable apoptotic cells in the A549DDP cells. When treatment with DDP and wortmannin, $8.9 \%$ of the apoptosis cells was detected $(P<0.05)$, when combined with U0126, 9.4\% of the apoptosis cells was detected $(P<0.05)$, however, when cells were treated with DDP and in combination with wortmannin and U0126, a synergistic cytotoxicity that $19.6 \%$ of the apoptosis cells was detected $(P<0.01)$ (Fig. 6B). This potentiated cytotoxicity by double pathway blockage is much higher than that of individual blockage of either pERK1/2 or pAKT. Similar results were also shown in the cellular viability inhibition (data not shown).

\section{Discussion}

This development of DDP resistance is a complex phenomenon involving multiple alterations. It can be intrinsic owing to germ-line genetic variation or acquired through altered mRNA or protein expression in key pharmacokinetic or pharmacodynamic pathways. It can also be the reduction of intracellular drug accumulation, increased DNA damage repair, and up-regulation of antiapoptotic genes [38, 39]. Moreover, activation of survivalassociated signal transduction pathways influences the cellular response to DDP treatment [40]. The crucial point in these events may be determined by signaling events downstream of the drug-DNA interactions. Acquisition of resistance by tumor cells to anticancer drugs may involve modifications in the cells' ability to trigger downstream signaling events. It is thus the balance between the various proteins present in the cell that finally decides whether the cell should live or die.

Recent focus has turned to clusterin (CLU) as a key contributor to chemoresistance to anticancer agents. Its role has been documented in prostate cancer for paclitaxel/ docetaxel resistance [41] as well as in renal [42], and melanoma [43],breast tumor cells [9, 44]. Most significantly, sCLU expression is documented to lead to broad-based resistance to other unrelated chemotherapeutic agents such as doxorubicin $[9,45]$, DDP $[9,45,46]$, and etoposide [47].

In the present study, we found DDP treatment increased the levels of sCLU in the A549 cells which made the A549 cells acquire the resistance to DDP. Overexpression of sCLU by sCLU transfection protected human lung cancer cells from DDP -induced cytotoxicity. In addition, inhibition of sCLU by shRNA to sCLU transfection could restore DDP sensitivity in DDP-sensitive A549 and resistant A549 ${ }^{\text {DDP }}$ cells. Although the involvement of sCLU acquires resistance to DDP in A549 cells, however, the molecular mechanisms underlying the effect of sCLU on lung cancer cell chemosensitivity is not known.

ERK1/2 activation results in the phosphorylation of many intracellular proteins that regulate various cellular functions ranging from proliferation and differentiation to apoptosis $[48,49]$. DDP can activate ERK1/2 in various cell types. Some studies have shown that activation of ERK1/2 is associated with an increase in cell survival in DDPtreated cells $[50,51]$. Accumulating evidence has indicated that the acquisition of resistance to chemotherapeutic drugs also involves the activation of the PI3K/AKT pathway [52]. Combining PI3K/AKT inhibitors with standard chemotherapy has been successful in increasing the efficacy of chemotherapeutic agents both in vivo and in vitro $[53,54]$.

Our previous studies have reported [33] that DDP activate both pERK1/2 and pAKT in A549 cells. Blockade of pERK1/2 or pAKT pathway alone with chemical inhibitors could moderately sensitiz A549 cells to DDP -induced apoptosis and reduce cell viability. Strikingly, much more effective potentiation of cytotoxicity to DDP was achieved when pERK1/2 and pAKT were concurrently blocked in vitro and vivo. Our study demonstrated that DDP-induced ERK1/2 and AKT activation participates in protection from DDP-mediated cytotoxic effect in A549 cells.

PI3K/AKT and ERK1/2 is believed to act downstream of sCLU [16-17, 34-36]. In this study, DDP could up-regulate the expression of sCLU, which is correlated with the activation of pAKT and pERK1/2. The fungal metabolite wortmannin and the synthetic compound 
LY294002 are the two currently known inhibitors that show fairly high specificity for PI3K. Wortmannin binds to the p110 catalytic subunit of PI3K, noncompetitively and irreversibly inhibiting (IC50, 2-4 nM) the enzyme [55]. It has been shown that wortmannin at $100 \mathrm{nM}$ causes $95 \%$ inhibition of PI3K and that the concentration required to induce apoptosis of cells maintained in growth factors correlates closely with that required for PI3K inhibition [56]. In the present study, we found that when pAKT protein was inhibited by wortmannin treatment, DDP could not induce pAKT upregulation, though sCLU was induced by DDP. In the A549DDP cells and A549/shRNA-sCLU cells, pAKT was also activated. However, when sCLU was inhibited by shRNA transfection, pAKT was significantly inhibited. Therefore, we suggest that pAKT was regulated by sCLU.

The mitogen-activated protein kinases (MAPKs) are serine/threonine kinases involved in the regulation of various cellular responses, such as cell proliferation, differentiation, and apoptosis [57]. The extracellular signal-regulated kinase (ERK) is a subfamily member of MAPKs, which is activated by an upstream kinase called MAPK/ERK kinase (MEK) in response to growth stimuli. The ERK pathway mediates a number of cellular fates including growth, proliferation, and survival [58].The ERK activation could be inhibited by U0126, a specific inhibitor of ERK upstream MAPK/ERK kinase (MEK). We demonstrated in the study that when pERK1/2 protein was prevented by U0126, cisplatin DDP could not induce pERK1/2 upregulation, though sCLU was induced by DDP. In the DDP resistant A549 ${ }^{\text {DDP }}$ cells and sCLU stably transfected A549 cells, pERK1/2 was also activated. However, when sCLU was inhibited by shRNA transfection, pERK1/2 was inhibited. Therefore, we suggest that pERK1/2 was regulated by sCLU.

We found in the study when the A549 cells were treated with wortmannin or U 0126 before treating with DDP, pERK1/2 and pAKT protein expression was inhibited. However, pAKT (pERK1/2) was independent of pERK1/2(pAKT)(data not shown), which was indicated that there was no relation between AKT and ERK in the study.

We also found in the study, sCLU was overexpressed in the A549DDP cells and A549/ shRNA-sCLU cells. In addition, inhibition of pERK1/2 or pAKT alone could in part sensitize the two cells to DDP treatment. However, when pERK1/2 and pAKT were concurrently blocked, significant recovery in chemosensitivity to DDP in A549DDP cells and A549/shRNAsCLU cells. Therefore, we suggest that sCLU protects lung cancer cells from DDP-induced cytotoxicity, which might be by activation of ERK1/2 and AKT. We have provided evidence here suggesting that sCLU signals regulate activation of ERK1/2 and AKT. However, it remains to be determined how sCLU links to ERK and AKT activation. Relatively little is known about the upstream signalling events that regulate ERK and AKT function in cancer cells. More investigation is needed to delineate the signalling mechanism underlying the ERK and AKT activation by sCLU.

Taken together, activation of sCLU may contribute to DDP resistance. Our results identify a new mechanism showing that the sCLU-AKT and sCLU- ERK1/2 signaling pathway is responsible for DDP resistance and suggest that targeting the SCLU-AKT and sCLU-ERK1/2 signaling pathway may overcome DDP resistance in human lung cancer.

\section{References}

Spira A, Ettinger DS: Multidisciplinary management of lung cancer. N Engl J Med 2004;350:379-392.

Neal JW, Gubens MA, Wakelee HA: Current management of small cell lung cancer. Clin Chest Med

2011;32:853-863.

3 Chang A: Chemotherapy, chemoresistance and the changing treatment landscape for NSCLC. Lung Cancer 2011;71:3-10.

4 Kartalou M, Essigmann JM: Mechanisms of resistance to cisplatin. Mutat Res 2001;478:23-43.

5 Rabik CA, Dolan ME: Molecular mechanisms of resistance and toxicity associated with platinating agents. Cancer Treat Rev 2007;33:9-23. 
6 Siddik ZH: Cisplatin: mode of cytotoxic action and molecular basis of resistance. Oncogene 2003;22:72657279.

7 Rosenberg ME, Silkensen J:Clusterin: physiologic and pathophysiologic considerations. Int J Biochem Cell Biol 1995;27:633-645.

$>8$ Jones SE, Jomary C: Clusterin. Int J Biochem Cell Biol 2002;34:427-431.

-9 Niu ZH, Wang Y, Chun B, Li CX, Wu L: Secretory clusterin (sCLU) overexpression is associated with resistance to preoperative neoadjuvant chemotherapy in primary breast cancer. Eur Rev Med Pharmacol Sci 2013;17:1337-1344.

10 Zoubeidi A, Chi K, Gleave M: Targeting the cytoprotective chaperone, clusterin, for treatment of advanced cancer.Clin Cancer Res 2010;16:1088-1093.

11 Yang GF, Li XM, Xie D: Overexpression of clusterin in ovarian cancer is correlated with impaired survival. Int J Gynecol Cancer 2009;19:1342-1346.

12 He LR, Liu MZ, Li BK, Rao HL, Liao YJ, Zhang LJ, Guan XY, Zeng YX, Xie D: Clusterin as a predictor for chemoradiotherapy sensitivity and patient survival in esophageal squamous cell carcinoma. Cancer Sci 2009;100:2354-2360.

13 Xie MJ, Motoo Y, Su SB, Mouri H, Ohtsubo K, Matsubara F, Sawabu N: Expression of clusterin in human pancreatic cancer. Pancreas 2002;25:234-238.

14 Park DC, Yeo SG, Wilson MR, Yerbury JJ, Kwong J, Welch WR, Choi YK, Birrer MJ, Mok SC, Wong KK: Clusterin interacts with Paclitaxel and confer Paclitaxel resistance in ovarian cancer. Neoplasia 2008;10:964-972.

15 Wang Y, Wang X, Zhao H, Liang B, Du Q: Clusterin confers resistance to TNF-alpha-induced apoptosis in breast cancer cells through NF-kappaB activation and Bcl-2 overexpression. J Chemother 2012;24:348-357.

-16 Chou TY, Chen WC, Lee AC, Hung SM, Shih NY, Chen MY: Clusterin silencing in human lung adenocarcinoma cells induces a mesenchymal-to-epithelial transition through modulating the ERK/Slug pathway. Cell Signal 2009;21:704-711.

17 Yan Y, Luo K, Zhang H, Chai W: RNA interference-mediated secretory clusterin gene silencing inhibits proliferation and promotes apoptosis of human non-small cell lung cancer cells. Hepatogastroenterology 2013;60:70-75.

18 Cheng CY, Cherng SH, Wu WJ, Yang TY, Huang XY, Liao FT, Wu MF, Sheu GT: Regulation of chemosensitivity and migration by clusterin in non-small cell lung cancer cells. Cancer Chemother Pharmacol 2012;69:145154.

19 Laskin JJ, Nicholas G, Lee C, Gitlitz B, Vincent M, Cormier Y, Stephenson J, Ung Y, Sanborn R, Pressnail B, Nugent F, Nemunaitis J, Gleave ME, Murray N, Hao D: Phase I/II trial of custirsen (OGX-011), an inhibitor of clusterin, in combination with a gemcitabine and platinum regimen in patients with previously untreated advanced non-small cell lung cancer. J Thorac Oncol 2012;7:579-586.

20 Cao C, Shinohara ET, Li H, Niermann KJ, Kim KW, Sekhar KR, Gleave M, Freeman M, Lu B: Clusterin as a therapeutic target for radiation sensitization in a lung cancer model. Int J Radiat Oncol Biol Phys 2005;63:1228-1236.

21 Kapoor S: Clusterin inhibition to enhance tumor chemosensitivity in systemic tumors. Cancer Chemother Pharmacol 2013;71:1101.

-22 Bortul R, Tazzari PL, Billi AM, Tabellini G, Mantovani I, Cappellini A, Grafone T, Martinelli G, Conte R, Martelli AM, Deguelin A: PI3K/AKT inhibitor, enhances chemosensitivity of leukaemia cells with an active PI3K/AKT pathway. Br J Haematol 2005;129:677-686.

23 Liu D, Zhang Y, Dang C, Ma Q, Lee W, Chen W: siRNA directed against TrkA sensitizes human pancreatic cancer cells to apoptosis induced by gemcitabine through an inactivation of PI3K/Akt-dependent pathway. Oncol Rep 2007;18:673-677.

24 Guinea Viniegra J, Hernández Losa J, Sánchez-Arévalo VJ, Parada Cobo C, Fernández Soria VM, Ramón y Cajal S, Sánchez-Prieto R: Modulation of PI3K/Akt pathway by E1a mediates sensitivity to cisplatin. Oncogene 2002;21:7131-7136.

25 McCubrey JA, Steelman LS, Abrams SL: Roles of the RAF/MEK/ERK and PI3K/PTEN/AKT pathways in malignant transformation and drug resistance. Adv Enzyme Regul 2006;46:249-279.

-26 McCubrey JA, Steelman LS, Chappell WH: Roles of the Raf/MEK/ERK pathway in cell growth, malignant transformation and drug resistance. Biochim Biophys Acta 2007;1773:1263-1284.

27 Sridhar SS, Hedley D, Siu LL: Raf kinase as a target for anticancer therapeutics. Mol Cancer Ther 2005;4:677-685. 


\section{Cellular Physiology $\quad$ Cell Physiol Biochem 2014;33:1162-1175

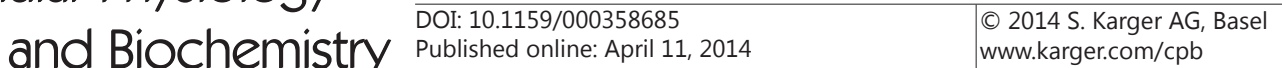 \\ Zhang et al.: Secreted Clusterin and Chemosensitivity in A549 Cells}

28 Tang Y, Liu F, Zheng C, Sun S, Jiang Y: Knockdown of clusterin sensitizes pancreatic cancer cells to gemcitabine chemotherapy by ERK1/2 inactivation. J Exp Clin Cancer Res 2012;31:73.

29 Si H, Peng C, Li J, Wang X, Zhai L, Li X, Li J: RNAi-mediated knockdown of ERK1/2 inhibits cell proliferation and invasion and increases chemosensitivity to cisplatin in human osteosarcoma U2-OS cells in vitro. Int J Oncol 2012;40:1291-1297.

-30 Zhou L, Luan H, Liu Q, Jiang T, Liang H, Dong X, Shang H: Activation of PI3K/Akt and ERK signaling pathways antagonized sinomenine-induced lung cancer cell apoptosis. Mol Med Rep 2012;5:1256-1260.

-31 Li H, Schmid-Bindert G, Wang D, Zhao Y, Yang X, Su B, Zhou C: Blocking the PI3K/AKT and MEK/ERK signaling pathways can overcome gefitinib-resistance in non-small cell lung cancer cell lines. Adv Med Sci 2011;56:275-284.

-32 Lee HY, Oh SH, Suh YA, Baek JH, Papadimitrakopoulou V, Huang S, Hong WK: Response of non-small cell lung cancer cells to the inhibitors of phosphatidylinositol 3-kinase/Akt- and MAPK kinase 4/c-Jun NH2terminal kinase pathways: an effective therapeutic strategy for lung cancer. Clin Cancer Res 2005;11:60656074.

33 Wang M, Liu ZM, Li XC, Yao YT, Yin ZX: Activation of ERK1/2 and Akt is associated with cisplatin resistance in human lung cancer cells. J Chemother 2013;25:162-169.

- 34 Li J, Jia L, Zhao P, Jiang Y, Zhong S, Chen D: Stable knockdown of clusterin by vector based RNA interference in a human breast cancer cell line inhibits tumour cell invasion and metastasis. J Int Med Res 2012;40:545555.

35 Xiu P, Dong X, Dong X, Xu Z, Zhu H, Liu F, Wei Z, Zhai B, Kanwar JR, Jiang H, Li J, Sun X: Secretory clusterin contributes to oxaliplatin resistance by activating Akt pathway in hepatocellular carcinoma. Cancer Sci 2013;104:375-382.

-36 Ma X, Bai Y: IGF-1 activates the P13K/AKT signaling pathway via upregulation of secretory clusterin. Mol Med Rep 2012;6:1433-1437.

-37 Scaltriti M, Bettuzzi S, Sharrard RM, Caporali A, Caccamo AE, Maitland NJ: Clusterin in both malignant and nonmalignant prostate epithelial cells induces cell cycle arrest and apoptosis. Br J Cancer 2004;91:18421850.

-38 Manic S, Gatti L, Carenini N, Fumagalli G, Zunino F, Perego P: Mechanisms controlling sensitivity to platinum complexes: role of p53 and DNA mismatch repair. Curr Cancer Drug Targets 2003;3:21-29.

-39 Siddik ZH: Cisplatin: mode of cytotoxic action and molecular basis of resistance. Oncogene 2003;22:72657279.

40 Hanahan D, Weinberg RA: The hallmarks of cancer. Cell 2000;100:57-70.

41 Springate CM, Jackson JK, Gleave ME, Burt HM: Efficacy of an intratumoral controlled release formulation of clusterin antisense oligonucleotide complexed with chitosan containing paclitaxel or docetaxel in prostate cancer xenograft models. Cancer Chemother Pharmacol 2005;56:239-247.

42 Miyake H, Hara S, Zellweger T, Kamidono S, Gleave ME, Hara I: Acquisition of resistance to Fas-mediated apoptosis by overexpression of clusterin in human renal-cell carcinoma cells. Mol Urol 2001;5:105-111.

-43 Hoeller C, Pratscher B, Thallinger C, Winter D, Fink D, Kovacic B, Sexl V, Wacheck V, Gleave ME, Pehamberger H, Jansen B: Clusterin regulates drug-resistance in melanoma cells. J Invest Dermatol 2005;124:1300-1307.

-44 Redondo M, Téllez T, Roldan MJ, Serrano A, García-Aranda M, Gleave ME, Hortas ML, Morell M: Anticlusterin treatment of breast cancer cells increases the sensitivities of chemotherapy and tamoxifen and counteracts the inhibitory action of dexamethasone on chemotherapy-induced cytotoxicity. Breast Cancer Res 2007;9:R86.

45 Lourda M, Trougakos IP, Gonos ES: Development of resistance to chemotherapeutic drugs in human osteosarcoma cell lines largely depends on up-regulation of Clusterin/Apolipoprotein J. Int J Cancer 2007;120:611-622.

-46 Karaca B, Atmaca H, Bozkurt E, Kisim A, Uzunoglu S, Karabulut B, Sezgin C, Sanli UA, Uslu R: Combination of AT-101/cisplatin overcomes chemoresistance by inducing apoptosis and modulating epigenetics in human ovarian cancer cells. Mol Biol Rep 2013;40:3925-3933.

-47 Pajak B, Orzechowski A: Ethylenediaminetetraacetic acid affects subcellular expression of clusterin protein in human colon adenocarcinoma COLO 205 cell line. Anticancer Drugs 2007;18:55-63.

48 Peyssonnaux C, Eychène A: The Raf/MEK/ERK pathway: new concepts of activation. Biol Cell 2001;93:5362. 
49 Zhuang S, Schnellmann RG: A death-promoting role for extracellular signal-regulated kinase. J Pharmacol Exp Ther 2006;319:991-997.

-50 Ko JC, Tsai MS, Chiu YF, Weng SH, Kuo YH, Lin YW: Up-regulation of extracellular signal-regulated kinase 1/2-dependent thymidylate synthase and thymidine phosphorylase contributes to cisplatin resistance in human non-small-cell lung cancer cells. J Pharmacol Exp Ther 2011;338:184-194.

-51 Gozdz A, Habas A, Jaworski J, Zielinska M, Albrecht J, Chlystun M, Jalili A, Hetman M: Role of N-methylD-aspartate receptors in the neuroprotective activation of extracellular signal-regulated kinase $1 / 2$ by cisplatin. J Biol Chem 2003;278:43663-43671.

-52 Huang WC, Hung MC: Induction of Akt activity by chemotherapy confers acquired resistance. J Formos Med Assoc 2009;108:180-194.

-53 Seo JH, Jeong ES, Lee KS, Heo SH, Jeong DG, Choi YK: Lentivirus-mediated shRNA targeting of cyclin D1 enhances the chemosensitivity of human gastric cancer to 5-fluorouracil. Int J Oncol 2013;43:2007-2014.

-54 Heavey S, O'Byrne KJ, Gately K: Strategies for co-targeting the PI3K/AKT/mTOR pathway in NSCLC. Cancer Treat Rev 2013:S0305-7372:00171-00180.

-55 Powis G, Bonjouklian R, Berggren MM, Gallegos A, Abraham R, Ashendel C, Zalkow L, Matter WF, Dodge J, Grindey G: Wortmannin, a potent and selective inhibitor of phosphatidylinositol-3-kinase. Cancer Res 1994;54:2419-2423.

-56 Yao R, Cooper GM: Requirement for phosphatidylinositol 3-kinase in the prevention of apoptosis by nerve growth factor. Nature (Lond) 1995;267:2003-2006.

57 Cross TG, Scheel-Toellner D, Henriquez NV, Deacon E, Salmon M, Lord JM: Serine/threonine protein kinases and apoptosis. Exp Cell Res 2000;256:34-41.

58 Cobb MH: MAP kinase pathways. Prog Biophys Mol Biol 1999;71:479-500. 